\title{
Organizing Pneumonia due to Actinomycosis: An Undescribed Association
}

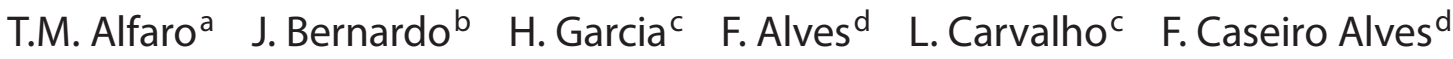 \\ C. Robalo Cordeiro ${ }^{\mathrm{a}}$ \\ ${ }^{a}$ Centre of Pulmonology of Coimbra University Medical School, ${ }^{b}$ Cardiothoracic Surgery Center, ${ }^{c}$ Pathological \\ Anatomy Department, and ${ }^{\mathrm{d}}$ Radiology Department, University Hospital of Coimbra, Coimbra, Portugal
}

\section{Established Facts}

- Organizing pneumonia is a pulmonary histological pattern that can be either cryptogenic or a response to various insults.

\section{Novel Insights}

- Actinomycosis, a rare infectious disease, can present itself with this pattern and should therefore be part of the differential diagnosis of organizing pneumonia.

\section{Key Words}

Actinomycosis $\cdot$ Interstitial lung diseases $\cdot$ Organizing pneumonia $\cdot$ Pulmonary inflammation

\begin{abstract}
Organizing pneumonia is a pathologic entity characterized by intra-alveolar buds of granulation tissue that can extend to the bronchiolar lumen. It is a non-specific finding reflecting a pattern of pulmonary response to aggression that can be cryptogenic or associated with several causes. Pulmonary actinomycosis is a rare infectious disease, of bacterial aetiology, and of difficult diagnosis. This disease usually causes non-specific respiratory symptoms and radiological findings, and the treatment is based on the use of antibiotics. The authors describe a clinical case of a 53-year-old male smoker
\end{abstract}

(50 pack years), initially seen for complaints of right-sided chest pain and sub-febrile temperature. Imaging studies revealed a mass in the inferior right lobe and enlarged mediastinal lymph nodes. Empirical treatment with antibiotics caused partial and temporary improvement. Transthoracic biopsy revealed a pattern of organizing pneumonia with giant multinucleated cell granulomas. Repeat imaging studies revealed an enlargement of the pulmonary mass and therefore a right inferior lobectomy was performed. The pathologic study revealed a histological pattern of organizing pneumonia surrounding inflammatory bronchiectasis with a large number of Actinomyces colonies. To our knowledge there is presently no report in the literature of organizing pneumonia associated with Actinomyces infection.

Copyright $\odot 2010$ S. Karger AG, Basel

\section{KARGER}

Fax +4161306 1234

E-Mail karger@karger.ch

www.karger.com
(C) 2010 S. Karger AG, Basel

0025-7931/11/0815-0433\$38.00/0

Accessible online at:

www.karger.com/res
Tiago M. Alfaro

Hospitais da Universidade de Coimbra

Serviço de Pneumologia, Praceta Dr. Mota Pinto

PT-3004-561 Coimbra (Portugal)

Tel. +351 966579 124, E-Mail alfarotm@gmail.com 


\section{Introduction}

Organizing pneumonia (OP) describes a histopathologic pattern characterized by the development of anastomosing plugs (buds) of granulation tissue, consisting of proliferating fibroblasts and myofibroblasts embedded in connective tissue, filling the distal pulmonary airspaces (alveoli and alveolar ducts) [1]. Mild interstitial inflammation and foamy macrophages in the non-filled alveoli are also typical [2]. The buds can also involve bronchiolar lumina (bronchiolitis obliterans), and in the past this entity was also called 'bronchiolitis obliterans organizing pneumonia'. However, currently the preferred designation is OP, to prevent confusion with other histological patterns [3] as, from a histological point of view, the pathology mainly concerns alveoli rather than bronchioles and the pulmonary function test findings reveal a restrictive rather than obstructive pattern.

This finding is a form of pulmonary response to a variety of insults and is not specific to a determinate disease. It can be related to infectious disease, drug induced injury [4], connective tissue disease [5], transplants, malignancy, post-radiotherapy (outside the radiation ports), inhalational injury, and vaccination. When extensive investigation fails to determine a cause in a patient with this pulmonary lesion, the diagnosis is then cryptogenic organizing pneumonia, a disease with variable clinical and radiological manifestations that usually responds in a favourable way to steroids [6-10].

Actinomycosis is a rare infectious disease caused by bacteria from the genus Actinomyces. These are Grampositive, facultative anaerobic, filamentous organisms, and can be found in the normal flora of the oral cavity, bronchial secretions and gastro-intestinal tract. The disease is usually caused by Actinomyces israelii, although other species may be involved [11]. The cervicofacial area is the most frequent site of involvement, but almost any organ can be affected. Pulmonary involvement occurs in about $15 \%$ of the cases and is therefore a very rare finding [12].

The disease occurs in all ages, and is more frequent in men. Alcoholism, underlying respiratory disease (chronic bronchitis, emphysema and bronchiectasis) and poor dental hygiene and dental disease are the known risk factors. Clinical features have changed in the last decades, presumably due to better overall hygiene and widespread availability of antibiotics. Presently, the usual presentation is of a slowly progressing pneumonia, with fever, weight loss, productive cough, and chest pain. Radiographic manifestations are also non-specific, unless there is direct involvement of the chest wall, with pleural involvement and bone destruction $[13,14]$. Confusion with tuberculosis and malignancy is common, but the infection may also be associated with neoplastic lesions, which should therefore be excluded [15].

The diagnosis of actinomycosis is usually a difficult one, with most patients being investigated for other diseases, before the diagnosis is made. Definitive diagnosis is made by culture of the bacteria in anaerobiosis, but often a presumptive diagnosis is made by histological observation of the characteristic sulphur granules [16]. The clinical material can be obtained by transthoracic or transbronchial biopsy though surgical biopsy is also frequently performed, mostly due to the suspicion of cancer.

Without treatment, actinomycosis can be fatal, but correct diagnosis provides the opportunity to institute treatment with long-term antibiotherapy, usually penicillin or its derivatives $[17,18]$.

\section{Case Report}

A 53-year-old male was seen in the outpatient clinic for complaints of right-side chest pain and mild fever of 2 weeks duration. He also complained of chronic cough and sputum, mostly in the morning, without any recent changes. He denied shortness of breath, headache, excessive sudoresis or other complaints. His pain was not related to movement or exercise.

Past medical history and habits included smoking (50 pack years) and a kidney stone several years ago.

He was self-medicating with paracetamol for the pain and fever with good results.

Examination showed a middle-aged man in good general condition, and no respiratory distress. His vital signs were normal. Oropharingeal examination revealed a dental prosthesis and several dental caries. Cardiac auscultation did not reveal any abnormalities, and on pulmonary auscultation scattered ronchi and crackles were present on the lower right pulmonary field. There was no peripheral oedema.

He was medicated with antibiotics for presumed low respiratory tract infection and discharged with an order for a chest X-ray.

Six weeks later, on a follow up visit he mentioned only temporary and partial improvement with the treatment and was now also complaining of cough and bloody sputum. Examination was unchanged. The chest X-ray, revealed an obliteration of the right cardio-phrenic angle, and was followed by chest CT, which showed a mass in the posterior right lower lobe of $6.6 \times 3.3 \mathrm{~cm}$, soft-tissue density and also right para-tracheal and subcarinal enlarged lymph nodes.

A transthoracic biopsy was performed to exclude cancer and histological study revealed a pattern of organizing pneumonia with giant multinucleated cell granulomas (fig. 1).

The patient was then referred for bronchoscopy which revealed no abnormal findings and microbiological (direct exam and culture) and cytological studies were negative. 


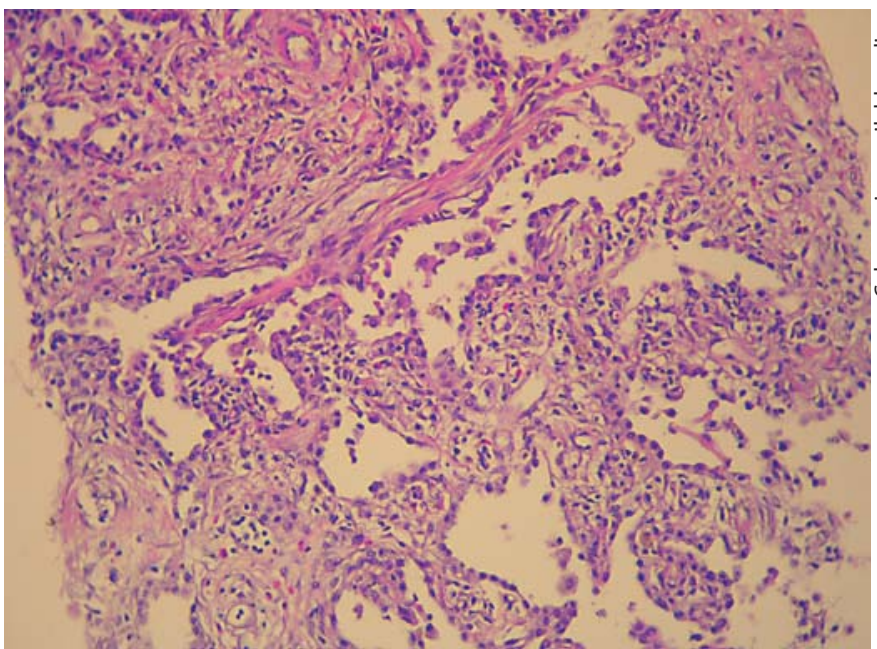

Fig. 1. Pulmonary trans-thoracic biopsy showing predominant myofibroblastic polyps occupying bronchiolar and alveolar spaces, interstitial uniform inflammation and fibroblast proliferation, associated with various multinucleated giant cell granulomas. $\mathrm{HE}, \times 200$.

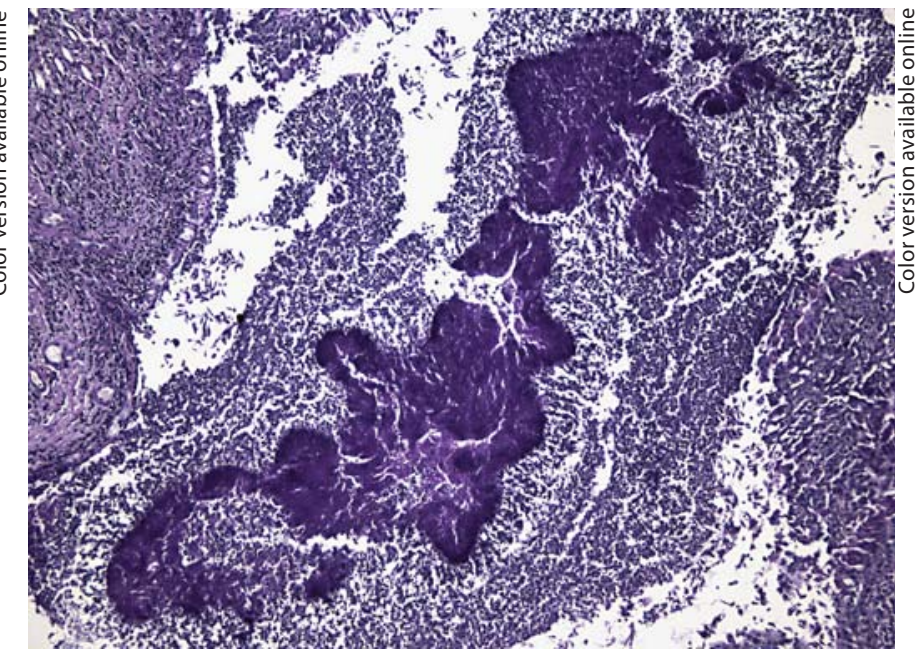

Fig. 3. Lower lobe resection: extensive areas of organizing pneumonia surrounding an inflammatory bronchiectasis occupied by large actinomyces colonies. PAS, $\times 200$.

Fig. 2. Second chest CT showing a parenchymal heterogeneous opacity in the right lower lobe and a small associated pleural effusion.

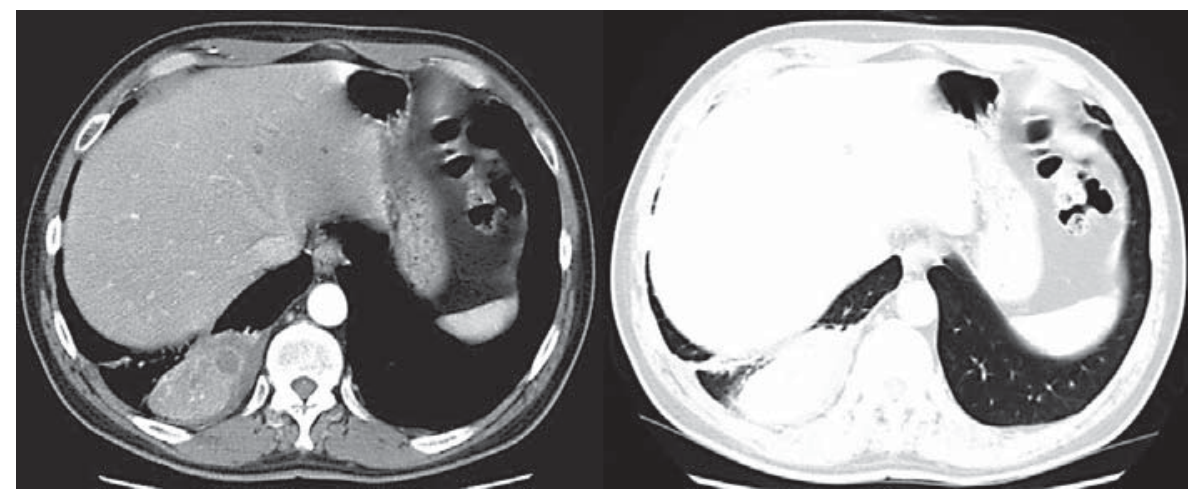

Electrocardiography showed sinus rhythm, and no signs of ischemic heart disease.

Lung function tests were remarkable for moderate obstruction with lung hyperinflation and a positive response to bronchodilators: FVC 2.831 (73.6\% predicted), $\mathrm{FEV}_{1} 2.001$ (64.2\% predicted), $\mathrm{FEV}_{1} / \mathrm{FVC} 70.64$, TLC 7.131 (115.3\% predicted), RV 3.491 (165.5\% predicted).

Due to the still high clinical suspicion of cancer or pulmonary tuberculosis, the patient was kept under close observation, but the complaints got progressively worse.

A repeat chest CT after 8 weeks revealed mass growth, now with $7 \times 6.8 \mathrm{~cm}$, the same enlarged lymph nodes and a small pleural effusion on the right side (fig. 2).

The decision was made to undertake a surgical approach. During surgery an inflammatory mass of $7-\mathrm{cm}$ diameter and with pleural adhesion was found and the surgeon decided to perform right inferior lobectomy with curative intent (no intra-operative pathology analysis was made).

Organizing Pneumonia due to

Actinomycosis
Histological analysis revealed extensive areas of organizing pneumonia, surrounding an inflammatory bronchiectasis with a large number of Actinomyces colonies (fig. 3). Due to the initial high suspicion of cancer, no microbiological analysis was made. After surgery the patient became asymptomatic.

\section{Discussion}

Pulmonary actinomycosis is rarely included in the differential diagnosis of a patient with a pulmonary infiltrate. The diagnosis is usually obtained by microbiological analysis of sterile fluid or by histological observation. The typical histological appearance includes a variable number of abscesses composed of actinomycotic granules (sulphur granules), surrounded by fibrosing granula- 
tion tissue. Giant cells may also be seen, and seem to be associated with aspiration [19]. The observed bronchiectasis in this patient's surgical biopsy argues in favour of this possibility.

Organizing pneumonia has been previously described in association with various bacterial infections, but not with actinomycosis. Although the clinical and imaging features of secondary organizing pneumonia are similar to cryptogenic organizing pneumonia, haemoptysis is uncommon in organizing pneumonia [1] and could be attributed in this case to the co-existant pulmonary actinomycosis [12]. The obstructive pattern found on the lung function tests is probably due to the patient's smoking history $[6,20]$. Histological exam alone is not enough to distinguish between cryptogenic and secondary OP [6, 21], although secondary OP may be associated with more interstitial inflammation or fibrosis and diffuse alveolar damage [1]. In the present case, the nature of the disease (unifocal infiltrate in a smoker with haemoptysis) led to a more invasive management option, with surgical excision, which is frequent in patients with focal OP [8]. This led to the diagnosis of an infection as the probable cause of the OP revealed by the trans-thoracic biopsy.

In conclusion, this case is remarkable not only for the development of pulmonary actinomycosis in an immunocompetent patient, but mostly for the associated histological appearance of organizing pneumonia, which was most likely caused by this infection. As far as the authors are aware this is the first report of organizing pneumonia in a patient with pulmonary actinomycosis. The differential diagnosis of this entity should therefore include this infection from now on.

\section{References}

$>1$ Cordier JF: Cryptogenic organising pneumonia. Eur Respir J 2006;28:422-446.

-2 Epler GR: Bronchiolitis obliterans organizing pneumonia. Arch Intern Med 2001;161: $158-164$.

-3 American Thoracic Society/European Respiratory Society International Multidisciplinary Consensus Classification of the Idiopathic Interstitial Pneumonias. Am J Respir Crit Care Med 2002;165:277-304.

-4 Tonelli AR, Lottenberg R, Allan RW, Sriram PS: Rituximab-induced hypersensitivity pneumonitis. Respiration 2009;78:225-229.

5 Shi JH, Liu HR, Xu WB, Feng RE, Zhang ZH, Tian XL, Zhu YJ: Pulmonary manifestations of Sjogren's syndrome. Respiration 2009;78: 377-386.

6 Oymak FS, Demirbas HM, Mavili E, Akgun H, Gulmez I, Demir R, Ozesmi M: Bronchiolitis obliterans organizing pneumonia: clinical and roentgenological features in 26 cases. Respiration 2005;72:254-262.

-7 Perez de Llano LA, Racamonde AV, Bande MJ, Piquer MO, Nieves FB, Feijoo AR: Bronchiolitis obliterans with organizing pneumonia associated with acute coxiella burnetiiinfection. Respiration 2001;68:425-427.
8 Maldonado F, Daniels CE, Hoffman EA, Yi ES, Ryu JH: Focal organizing pneumonia on surgical lung biopsy: causes, clinicoradiologic features, and outcomes. Chest 2007; 132:1579-1583.

\$9 Oikonomou A, Hansell DM: Organizing pneumonia: the many morphological faces. Eur Radiol 2002;12:1486-1496.

10 Schlesinger C, Koss MN: The organizing pneumonias: an update and review. Curr Opin Pulm Med 2005;11:422-430.

11 Kapotsis GE, Daniil Z, Klimopoulos S, Malagari K, Roussos C, Rontogianni D, Papiris SA: A painful and swollen right breast in a young male. Eur Respir J 2004;24:10661068

12 Mabeza GF, Macfarlane J: Pulmonary actinomycosis. Eur Respir J 2003;21:545-551.

13 Kim TS, Han J, Koh WJ, Choi JC, Chung MJ, Lee JH, Shim SS, Chong S: Thoracic actinomycosis: CT features with histopathologic correlation. AJR Am J Roentgenol 2006;186: 225-231.

14 Cheon JE, Im JG, Kim MY, Lee JS, Choi GM, Yeon KM: Thoracic actinomycosis: CT findings. Radiology 1998;209:229-233.

15 Madhusudhan KS, Gamanagatti S, Seith A, Hari S: Pulmonary infections mimicking cancer: report of four cases. Singapore Med J 2007;48:e327-e331.
16 de Montpreville VT, Nashashibi N, Dulmet EM: Actinomycosis and other bronchopulmonary infections with bacterial granules. Ann Diagn Pathol 1999;3:67-74.

17 Yildiz O, Doganay M: Actinomycoses and nocardia pulmonary infections. Curr Opin Pulm Med 2006;12:228-234.

-18 Takayanagi N, Kagiyama N, Ishiguro T, Tokunaga D, Sugita Y: Etiology and outcome of community-acquired lung abscess. Respiration 2010;80:98-105.

19 Brown JR: Human actinomycosis: a study of 181 subjects. Hum Pathol 1973;4:319-330.

20 Glaser S, Schaper C, Obst A, Ittermann T, Volzke H, Felix SB, Vogelmeier C, Dorr M, Ewert R, Koch B: Impact of different definitions of airflow limitation on the prevalence of chronic obstructive pulmonary disease in the general population. Respiration 2010;80: 292-300.

21 Bradley B, Branley HM, Egan JJ, Greaves MS, Hansell DM, Harrison NK, Hirani N, Hubbard R, Lake F, Millar AB, Wallace WA, Wells AU, Whyte MK, Wilsher ML: Interstitial lung disease guideline: the British thoracic society in collaboration with the thoracic society of Australia and New Zealand and the Irish thoracic society. Thorax 2008; 63(suppl 5):v1-v58. 